\title{
BMJ Open Association between human resources and risk of hospitalisation in end-stage renal disease outpatients receiving haemodialysis: a longitudinal cohort study using claim data during 2013-2014
}

\author{
Hoon-Hee Choi, ${ }^{1,2}$ Kyu-Tae Han, ${ }^{3,4}$ Chung Mo Nam, ${ }^{5}$ Ki Tae Moon, ${ }^{6}$ \\ Woorim Kim, ${ }^{3,4}$ Eun-Cheol Park ${ }^{4,5}$
}

To cite: Choi H-H, Han K-T, Nam CM, et al. Association between human resources and risk of hospitalisation in end-stage renal disease outpatients receiving haemodialysis: a longitudinal cohort study using claim data during 2013-2014. BMJ Open 2016;6: 011319. doi:10.1136/bmjopen-2016011319

- Prepublication history for this paper is available online. To view these files please visit the journal online (http://dx.doi.org/10.1136/ bmjopen-2016-011319).

Received 28 January 2016 Revised 19 July 2016 Accepted 29 July 2016

CrossMark

For numbered affiliations see end of article.

Correspondence to Dr Eun-Cheol Park; ecpark@yuhs.ac

\section{ABSTRACT}

Objective: The number of patients requiring haemodialysis has gradually increased in South Korea. Owing to this growth, concerns have been raised regarding haemodialysis quality of care, and healthcare professionals must consider alternatives for appropriate management of patients with chronic kidney disease (CKD). Therefore, we investigated the association between risk of hospitalisation of outpatients who received haemodialysis due to end-stage renal disease (ESRD) and the human resources of the haemodialysis unit.

Setting: We used data from National Health Insurance (NHI) claims during October 2013 to September 2014.

Participants: These data comprised 40543 outpatients with ESRD (4 751047 outpatient cases) who received haemodialysis.

Interventions: No interventions were made. Outcome measure: We performed Poisson regression analysis using a generalised estimating equation that included both patient and haemodialysis unit characteristics to examine the factors associated with hospitalisation of outpatients with ESRD.

Results: Among 4751047 outpatient cases, 27997 $(0.59 \%)$ were hospitalised during the study period. A higher proportion of haemodialysis patient care specialists and a higher number of nurses experienced in haemodialysis were inversely associated with the risk of hospitalisation (per 10\% increase in haemodialysis patient care specialists: relative risk (RR) $=0.987,95 \% \mathrm{Cl} 0.981$ to 0.993 ; per 10-person increase in nurses who provided haemodialysis: $\mathrm{RR}=0.876,95 \% \mathrm{Cl} 0.833$ to 0.921 ). In addition, such associations were greater in severe patients.

Conclusions: Our findings suggest that haemodialysis units with high-quality, haemodialysisspecialised human resources could positively affect the outcomes of outpatients with ESRD. Based on our findings, health policymakers and professionals should implement strategies for the optimal management of patients with CKD.

\section{Strengths and limitations of this study}

- Our results may prove useful for designing an effective strategy for managing patients with CKD receiving haemodialysis.

- This study reflects the variety and severity of each patient and the medical institution situation.

- We were not able to include other factors that could affect outcome variables in this study as the data used were secondary data based on the NHI claim data.

- We could not identify whether each patient actually received treatment from specific human resources in each haemodialysis unit.

\section{INTRODUCTION}

Since the overall health status of South Koreans has improved due to economic and health technology development during the late 20th century, the elderly population has grown, and South Korea is expected to become an aged society. ${ }^{1}$ Naturally, health problems related to ageing, such as chronic diseases, have become more prevalent compared with past centuries, leading to a gradual increase in the usage of healthcare due to diabetes and hypertension, as well as problems related to such diseases (hypertension: $27.3 \%$ and diabetes: $7.7 \%$ among those $>30$ years of age in 2013). ${ }^{2}$ One of these related diseases is chronic kidney disease (CKD), which is defined as a progressive loss of kidney function and generally causes neurological, cardiovascular and digestive symptoms, as well as anaemia or haemorrhage, and in severe cases, death. ${ }^{3}$

Patients with CKD receive medical services for preventing comorbid conditions and progression of CKD, including haemodialysis, peritoneal dialysis and kidney transplantation, 
based on the severity of their CKD. ${ }^{4-6}$ Haemodialysis is a common treatment for severe cases of CKD. According to reports by the Health Insurance Review and Assessment Service (HIRA), the number of patients who underwent haemodialysis and the associated average medical cost due to haemodialysis have rapidly increased (56 896 patients in 2009 to 69837 in 2013; US\$1.1 billion in 2009 to US $\$ 1.4$ billion in 2013). ${ }^{7}$

Previous studies have found that several factors, such as workload, haemodialysis unit human resources and unit characteristics could reduce the quality of care in managing patients with CKD. ${ }^{8} 9$ Additionally, as the number of patients receiving haemodialysis increases, the quality of care in providing haemodialysis for CKD is expected to decrease due to the increasing workload. Although the South Korean government introduced healthcare quality assessment for haemodialysis unit resources to improve the quality of care when providing haemodialysis for patients with CKD after 2009, few studies have examined the relationship between haemodialysis unit resources and the quality of haemodialysis care after the introduction of healthcare quality assessment. ${ }^{10}$ Therefore, concerns remain with respect to optimal care and reduction in the quality of haemodialysis due to hospital competition and overcrowding. We thus focused only on patients with diagnosed end-stage renal disease (ESRD) who received haemodialysis and investigated which factors, including human resources, in each haemodialysis unit were associated with hospitalisation due to ESRD as indicators for quality of care. The results of this study provide important information regarding healthcare quality assessment for haemodialysis and may aid in providing solutions for possible future problems related to the care of patients with ESRD.

\section{MATERIALS AND METHODS}

\section{Data source and study population}

We used two data sets from the National Health Insurance (NHI) claim data. The first data set was claim data for 53583 patients previously diagnosed with CKD (International Classification of Diseases (ICD)-10: N18) who received haemodialysis at medical institutions during October 2013 to September 2014. Given that South Korea introduced the NHI after 1989, these patients could be identified based on the electronic data interchange claim code that was provided during reimbursement for healthcare services. The second data set included claim data regarding medical institution usage due to CKD during October 2008 to September 2013 and claim data regarding hospitalisation due to CKD during October 2012 to September 2013; this data set reflected the severity and duration of illness in patients with CKD receiving haemodialysis. These two data sets were merged for the final analysis to investigate the association between factors including human and medical resources in each haemodialysis unit and hospitalisation. We then excluded patients diagnosed with CKD stages
1-4 to reduce variation between patients and included only patients diagnosed with ESRD (ICD-10-CM: N18.6). Patients with illness durations of $<1$ year were also excluded to remove the possibility of including hospitalisation due to arteriovenous fistula formation for haemodialysis rather than to the worsening status of the patient. In addition, we excluded hospitals that did not meet the criteria for haemodialysis machines for patients with hepatitis B, as most hospitals met such criteria (unsatisfied: two hospitals). Ultimately, 4751047 outpatient cases of 40543 patients were included for analysis. The unit of analysis was outpatient cases due to haemodialysis. Since this study used secondary data from the NHI claim data, the requirement of informed consent was waived in the study, as the patient's information was anonymised and de-identified prior to analysis.

\section{Variables}

The outcome variable used in this study was whether patients who were previously diagnosed with ESRD were hospitalised by ESRD based on major diagnosis after receiving outpatient care due to haemodialysis. If a patient with ESRD was hospitalised after specific outpatient care for haemodialysis, we assumed that this outpatient care caused the hospitalisation due to the worsening status of the patient with ESRD receiving haemodialysis treatment. ${ }^{11} 12$

The exposures of interest in this study were the human resources at each medical institution, listed as follows: the total number of doctors who provided haemodialysis, the proportion of haemodialysis patient care specialists, the total number of nurses who provided haemodialysis and the proportion of nurses experienced in haemodialysis. The total number of doctors or nurses was defined as the actual number of doctors or nurses who provided haemodialysis services for patients with ESRD. The haemodialysis patient care specialists were defined as follows: (1) specialists who were trained as nephrologists among internal medicine or paediatric specialists; (2) specialists who were trained in haemodialysis for more than 1 year after training as internal medicine or paediatric specialists; (3) internal medicine or paediatric specialists who had experience in caring for patients with haemodialysis for more than 3 years. The proportion of haemodialysis patient care specialists was defined as the proportion of such specialists among the total number of doctors who provided the haemodialysis.

We also adjusted for patient and haemodialysis unit characteristics when analysing the relationship between human resources and hospitalisation after haemodialysis. The included patient characteristics were as follows: age, sex, type of insurance coverage, experience of prehospitalisation within 1 year, duration of illness and Charlson Comorbidity Index (CCI). Age was classified as $\leq 49$, $50-59,60-69$ and $\geq 70$ years. Two types of insurance coverage were considered, as defined by NHI: coverage for the general population and coverage for 
beneficiaries of medical aid (low-income, disabled and elderly patients, who are all provided with free inpatient and outpatient care by the government). Therefore, the type of insurance coverage could represent the socioeconomic status of each inpatient. Prehospitalisation within 1 year was defined as whether a patient was hospitalised due to ESRD during October 2012 to September 2013 to reflect the severity of each patient who was previously diagnosed with ESRD. The duration of illness was defined as the period from the first diagnosis of ESRD and was measured in years. We assumed that patients with a shorter duration of illness could not easily manage their status or were relatively more unstable. ${ }^{13}{ }^{14} \mathrm{CCI}$ was calculated using all comorbid conditions except CKD on hospitalisation. The data used in this study included the information for a maximum of 10 comorbidities excluding major diagnoses. This information was collected from previous outpatient or inpatient care or their comorbidities when each patient visited the hospital. These comorbid symptoms were weighted and scored with additional points added to consider comorbidities that could affect the outcomes of patients with ESRD.

The included haemodialysis unit characteristics (excluding human resources) included the type of medical institution, presence of a nephrologist, haemodialysis volume per doctor, number of beds, emergency equipment in the haemodialysis unit, fulfilment rate of criteria for duration of water analysis, number of haemodialysis machines and proportion of medical cost due to CKD. Medical institutions were classified as 'general hospital' or 'clinic or hospital'. The variable for emergency equipment in the haemodialysis unit merely indicated the presence of emergency equipment in the unit. The fulfilment rate of criteria for the duration of water analysis was based on whether each haemodialysis unit met the criteria for the frequency of the water analysis. The proportion of medical cost due to CKD was out of the total medical cost of each haemodialysis unit and was included to reflect the expertise in managing patients with CKD in each haemodialysis unit.

\section{Statistical analysis}

We examined the distribution of each categorical variable by examining their frequencies and percentages and then performed $\chi^{2}$ tests to investigate their association with hospitalisation after haemodialysis in patients diagnosed with ESRD. In addition, we performed an analysis of variance to compare the average values and SDs for continuous variables. In order to investigate the relationship between human resources in a haemodialysis unit and the risk of hospitalisation after haemodialysis in patients with ESRD, we performed a Poisson regression analysis using a generalised estimating equation (GEE) model. GEE models with link logit functions that included both patient and hospital characteristics were analysed, as the data used in this study were hierarchically structured and had binary outcome variables. Additionally, to examine the differences in the risk of hospitalisation, we performed subgroup analyses, adjusting both patient and hospital characteristics by the presence of a nephrologist, duration of illness and CCI. All statistical analyses were performed using SAS statistical software, V.9.2.

\section{RESULTS}

The data used in this analysis comprised 4751047 outpatient cases of 40543 patients. Table 1 shows the univariate associations between various independent variables including patient and haemodialysis unit characteristics and hospitalisation due to ESRD after haemodialysis. Among 4751047 outpatient cases, 27997 $(0.59 \%)$ were hospitalised due to ESRD during the study period. Those in the elderly group were more frequently hospitalised after haemodialysis than all other age groups. Outpatient cases among those with medical aid also more frequently involved hospitalisation than other cases (NHI $0.57 \%$, medical aid $0.65 \%$ ), and outpatient cases among those with prehospitalisation history within 1 year more frequently involved hospitalisation than other cases (yes $0.89 \%$, no $0.41 \%$ ). In addition, patients with a shorter duration of illness were more frequently hospitalised after haemodialysis than those with a longer duration of illness. Regarding haemodialysis unit characteristics, cases in general hospitals more frequently involved hospitalisation due to ESRD than cases in clinics or hospitals. Furthermore, cases in haemodialysis units with a nephrologist more frequently involved hospitalisation than cases in haemodialysis units without a nephrologist. The average number of total doctors in each haemodialysis unit was higher in hospitalisation cases, whereas the average number of total nurses, the proportion of haemodialysis patient care specialists and the proportion of nurses with haemodialysis experience were lower in hospitalisation cases. The average values of haemodialysis volume per doctor were also lower in hospitalisation cases. In addition, the average value for the proportion of medical cost due to CKD was lower in hospitalisation cases (table 1 ).

Table 2 shows the results of the Poisson regression analysis using the GEE model to investigate the relationship between human resources and risk of hospitalisation due to ESRD. The age of the patient correlated with the risk of hospitalisation due to ESRD. Additionally, an analysis by type of insurance coverage indicated that outpatient cases of medical aid beneficiaries were associated with the risk of hospitalisation due to ESRD more so than other types of insurance coverage (relative risk (RR) 1.334, 95\% CI 1.281 to 1.389 ; ref.=NHI). In addition, patients who experienced prehospitalisation within 1 year were associated with the risk of hospitalisation (RR 1.837, 95\% CI 1.773 to 1.903 ). CCI also tended to increase with the risk of hospitalisation due to ESRD, although this correlation was not statistically significant. Regarding haemodialysis unit characteristics, a higher proportion of haemodialysis patient care specialists and a higher number of total nurses who provided 
Table 1 General characteristics of the study population and haemodialysis units by hospitalisation after haemodialysis

\begin{tabular}{|c|c|c|c|c|c|}
\hline \multirow[b]{3}{*}{ Variables } & \multicolumn{4}{|c|}{ Hospitalisation after haemodialysis ( $N=4751$ 047) } & \multirow[b]{3}{*}{ p Value } \\
\hline & \multicolumn{2}{|c|}{ Yes } & \multicolumn{2}{|c|}{ No } & \\
\hline & N/mean & Per cent/SD & N/mean & Per cent/SD & \\
\hline \multicolumn{6}{|l|}{ Patient characteristics } \\
\hline \multicolumn{6}{|l|}{ Age (years) } \\
\hline$\leq 49$ & 4124 & 0.41 & 1011407 & 99.59 & $<0.0001$ \\
\hline $50-59$ & 6743 & 0.52 & 1278756 & 99.48 & \\
\hline $60-69$ & 7889 & 0.63 & 1241225 & 99.37 & \\
\hline$\geq 70$ & 9241 & 0.77 & 1191662 & 99.23 & \\
\hline \multicolumn{6}{|l|}{ Sex } \\
\hline Male & 15815 & 0.58 & 2719129 & 99.42 & 0.0003 \\
\hline Female & 12182 & 0.60 & 2003921 & 99.40 & \\
\hline \multicolumn{6}{|l|}{ Type of insurance coverage } \\
\hline $\mathrm{NHI}$ & 20734 & 0.57 & 3621256 & 99.43 & $<0.0001$ \\
\hline Medical aid & 7263 & 0.65 & 1101794 & 99.35 & \\
\hline \multicolumn{6}{|l|}{ Experience of prehospitalisation during 1 year } \\
\hline Yes & 15647 & 0.89 & 1743611 & 99.11 & $<0.0001$ \\
\hline No & 12350 & 0.41 & 2979439 & 99.59 & \\
\hline \multicolumn{6}{|l|}{ Duration of illness (years) } \\
\hline $2-3$ & 2816 & 0.73 & 381554 & 99.27 & $<0.0001$ \\
\hline $3-4$ & 3624 & 0.68 & 529343 & 99.32 & \\
\hline $4-5$ & 3154 & 0.65 & 483078 & 99.35 & \\
\hline $5-6$ & 3806 & 0.65 & 581142 & 99.35 & \\
\hline$>6$ & 14597 & 0.53 & 2747933 & 99.47 & \\
\hline \multicolumn{6}{|l|}{$\mathrm{CCl}$} \\
\hline 0 & 3351 & 0.42 & 797328 & 99.58 & $<0.0001$ \\
\hline 1,2 & 12579 & 0.58 & 2173291 & 99.42 & \\
\hline 3,4 & 9824 & 0.70 & 1385868 & 99.30 & \\
\hline $5+$ & 2243 & 0.61 & 366563 & 99.39 & \\
\hline \multicolumn{6}{|l|}{ Haemodialysis unit characteristics } \\
\hline \multicolumn{6}{|l|}{ Type of medical institution } \\
\hline General hospital (N=234) & 15137 & 0.82 & 1825112 & 99.18 & $<0.0001$ \\
\hline Clinic or hospital $(\mathrm{N}=395)$ & 12860 & 0.44 & 2897938 & 99.56 & \\
\hline \multicolumn{6}{|l|}{ Presence of nephrologist } \\
\hline Yes $(N=114)$ & 8815 & 0.79 & 1102400 & 99.21 & $<0.0001$ \\
\hline No $(\mathrm{N}=515)$ & 19182 & 0.53 & 3620650 & 99.47 & \\
\hline Total number of doctors who provided haemodialysis & 1.99 & \pm 1.57 & 1.83 & \pm 1.40 & $<0.0001$ \\
\hline Proportion of haemodialysis patient care specialists & 83.59 & \pm 33.80 & 84.10 & \pm 33.09 & 0.0107 \\
\hline Total number of nurses who provided haemodialysis & 10.84 & \pm 6.85 & 11.10 & \pm 7.04 & $<0.0001$ \\
\hline Proportion of nurses experienced in haemodialysis & 74.53 & \pm 17.09 & 75.22 & \pm 16.65 & $<0.0001$ \\
\hline Volume of haemodialysis per doctor & 1.77 & \pm 0.97 & 1.97 & \pm 1.06 & $<0.0001$ \\
\hline Number of beds & 330.98 & \pm 382.58 & 246.18 & \pm 375.48 & $<0.0001$ \\
\hline \multicolumn{6}{|l|}{ Emergency equipment in haemodialysis unit } \\
\hline Yes $(N=573)$ & 26483 & 0.59 & 4469487 & 99.41 & 0.1794 \\
\hline No $(N=56)$ & 1514 & 0.59 & 253563 & 99.41 & \\
\hline Fulfilment rate of criteria for duration of water analysis & 90.74 & \pm 19.81 & 90.31 & \pm 19.93 & 0.0004 \\
\hline Number of haemodialysis machines & 34.40 & \pm 18.18 & 34.36 & \pm 18.70 & 0.6967 \\
\hline Proportion of medical cost due to CKD & 37.85 & \pm 38.61 & 53.05 & \pm 40.71 & $<0.0001$ \\
\hline Total & 27997 & 0.59 & 4723050 & 99.41 & \\
\hline
\end{tabular}

$\mathrm{CCl}$, Charlson Comorbidity Index; CKD, chronic kidney disease; NHI, National Health Insurance.

haemodialysis were inversely associated with the risk of hospitalisation (proportion of haemodialysis patient care specialists, per $10 \%$ increase, RR $0.987,95 \%$ CI 0.981 to 0.993 ; number of total nurses who provided haemodialysis, RR $0.876,95 \%$ CI 0.833 to 0.921$)$. A higher proportion of medical cost due to CKD among total medical costs in each haemodialysis unit was inversely associated with the risk of hospitalisation (RR $0.924,95 \%$ CI 0.915 to 0.933 ; table 2).

We also performed a subgroup analysis to examine differences in relation to the risk of hospitalisation by duration of illness, CCI and presence of a nephrologist at the haemodialysis unit. A higher proportion of haemodialysis patient care specialists were more inversely associated with 
Table 2 Risk of hospitalisation after haemodialysis by patient and haemodialysis unit characteristics

\begin{tabular}{|c|c|c|c|}
\hline \multirow[b]{2}{*}{ Variables } & \multicolumn{3}{|c|}{ Hospitalisation after haemodialysis } \\
\hline & $\mathbf{R R}$ & $95 \% \mathrm{Cl}$ & p Value \\
\hline \multicolumn{4}{|l|}{ Patient characteristics } \\
\hline \multicolumn{4}{|l|}{ Age (years) } \\
\hline$\leq 49$ & 1.000 & - & - \\
\hline 50-59 & 1.205 & 1.090 to 1.332 & 0.0003 \\
\hline $60-69$ & 1.455 & 1.317 to 1.608 & $<0.0001$ \\
\hline$\geq 70$ & 1.732 & 1.560 to 1.921 & $<0.0001$ \\
\hline \multicolumn{4}{|l|}{ Sex } \\
\hline Male & 1.000 & 0.966 to 1.035 & 0.981 \\
\hline Female & 1.000 & - & \\
\hline \multicolumn{4}{|l|}{ Type of insurance coverage } \\
\hline $\mathrm{NHI}$ & 1.000 & - & - \\
\hline Medical aid & 1.334 & 1.281 to 1.389 & $<0.0001$ \\
\hline \multicolumn{4}{|l|}{ Experience of prehospitalisation within 1 year } \\
\hline Yes & 1.837 & 1.773 to 1.903 & $<0.0001$ \\
\hline No & 1.000 & - & - \\
\hline \multicolumn{4}{|l|}{ Duration of illness (years) } \\
\hline $2-3$ & 1.000 & - & - \\
\hline $3-4$ & 0.983 & 0.918 to 1.052 & 0.6158 \\
\hline $4-5$ & 0.987 & 0.917 to 1.063 & 0.7342 \\
\hline $5-6$ & 1.018 & 0.948 to 1.094 & 0.6185 \\
\hline$>6$ & 0.911 & 0.860 to 0.965 & 0.0016 \\
\hline \multicolumn{4}{|l|}{$\mathrm{CCl}$} \\
\hline 0 & 1.000 & - & - \\
\hline 1,2 & 1.058 & 0.955 to 1.173 & 0.2811 \\
\hline 3,4 & 1.065 & 0.956 to 1.186 & 0.2549 \\
\hline $5+$ & 1.104 & 0.982 to 1.241 & 0.0981 \\
\hline \multicolumn{4}{|l|}{ Haemodialysis characteristics } \\
\hline \multicolumn{4}{|l|}{ Type of medical institution } \\
\hline General hospital (N=234) & 1.000 & - & - \\
\hline Clinic or hospital $(\mathrm{N}=395)$ & 0.940 & 0.869 to 1.017 & 0.1220 \\
\hline \multicolumn{4}{|l|}{ Presence of nephrologist } \\
\hline Yes $(N=114)$ & 0.982 & 0.936 to 1.029 & 0.4429 \\
\hline No $(N=515)$ & 1.000 & - & \\
\hline Total number of doctors who provided haemodialysis (per 10-doctor increase) & 1.001 & 0.973 to 1.030 & 0.9541 \\
\hline Proportion of haemodialysis patient care specialists (per 10\% increase) & 0.987 & 0.981 to 0.993 & $<0.0001$ \\
\hline Total number of nurses who provided haemodialysis (per 10-nurse increase) & 0.876 & 0.833 to 0.921 & $<0.0001$ \\
\hline Proportion of nurses experienced in haemodialysis (per $10 \%$ increase) & 0.993 & 0.983 to 1.003 & 0.1576 \\
\hline Volume of haemodialysis per doctor & 0.963 & 0.936 to 0.992 & 0.0115 \\
\hline Number of beds (per 10-bed increase) & 0.999 & 0.998 to 1.000 & 0.1065 \\
\hline \multicolumn{4}{|l|}{ Emergency equipment in haemodialysis unit } \\
\hline Yes $(\mathrm{N}=573)$ & 1.000 & - & - \\
\hline No $(\mathrm{N}=56)$ & 0.930 & 0.856 to 1.011 & 0.0882 \\
\hline Fulfilment rate of criteria for duration of water analysis (per $10 \%$ increase) & 0.978 & 0.969 to 0.987 & $<0.0001$ \\
\hline Number of haemodialysis machines (per 10-machine increase) & 1.046 & 1.029 to 1.063 & $<0.0001$ \\
\hline Proportion of medical cost due to CKD (per 10\%) & 0.924 & 0.915 to 0.933 & $<0.0001$ \\
\hline
\end{tabular}

the risk of hospitalisation in the presence of a nephrologist at the haemodialysis unit; however, a higher number of total nurses were more inversely associated with the outcome variable in the absence of a nephrologist at the haemodialysis unit. By duration of illness, a higher proportion of haemodialysis patient care specialists and a higher number of total nurses who provided haemodialysis were inversely associated with the risk of hospitalisation in both groups, and the magnitude was also similar. Similar results were also obtained in the subgroup analysis by CCI, although a higher number of nurses were more inversely associated with the risk of hospitalisation in cases with a CCI of more than 3 (table 3 ).

\section{DISCUSSION}

Since the late 20th century, new problems related to chronic diseases have rapidly emerged in South Korea. 
Table 3 Subgroup analysis by presence of nephrologist, duration of illness and $\mathrm{CCl}^{*}$

\begin{tabular}{|c|c|c|c|c|}
\hline Subgroup & & Variables & RR & p Value \\
\hline \multirow{8}{*}{$\begin{array}{l}\text { Presence of } \\
\text { nephrologist }\end{array}$} & \multirow[t]{4}{*}{ Yes } & Total number of doctors who provided haemodialysis (per 10-doctor increase) & 1.023 & 0.2614 \\
\hline & & Proportion of haemodialysis patient care specialists (per $10 \%$ increase) & 0.970 & 0.0062 \\
\hline & & Total number of nurses who provided haemodialysis (per 10-nurse increase) & 0.901 & \\
\hline & & Proportion of nurses experienced in haemodialysis (per 10\% increase) & 0.991 & 0.360 \\
\hline & \multirow[t]{4}{*}{ No } & Total number of doctors who provided haemodialysis (per 10-doctor increase) & 0.980 & 0.3125 \\
\hline & & Proportion of haemodialysis patient care specialists (per $10 \%$ increase) & 0.989 & 0.0005 \\
\hline & & Total number of nurses who provided haemodialysis (per 10-nurse increase) & 0.864 & $<0.000$ \\
\hline & & Proportion of nurses experienced in haemodialysis (per $10 \%$ increase) & 0.994 & 0.3191 \\
\hline \multirow{8}{*}{$\begin{array}{l}\text { Duration of } \\
\text { illness (years) }\end{array}$} & \multirow[t]{4}{*}{$\leq 5$} & Total number of doctors who provided haemodialysis (per 10-doctor increase) & 1.016 & 0.4756 \\
\hline & & Proportion of haemodialysis patient care specialists (per $10 \%$ increase) & 0.986 & 0.0016 \\
\hline & & Total number of nurses who provided haemodialysis (per 10-nurse increase) & 0.880 & 0.000 \\
\hline & & Proportion of nurses experienced in haemodialysis (per $10 \%$ increase) & 0.996 & 0.559 \\
\hline & \multirow[t]{4}{*}{$6+$} & Total number of doctors who provided haemodialysis (per 10-doctor increase) & 0.986 & 0.449 \\
\hline & & Proportion of haemodialysis patient care specialists (per $10 \%$ increase) & 0.989 & 0.007 \\
\hline & & Total number of nurses who provided haemodialysis (per 10-nurse increase) & 0.879 & 0.000 \\
\hline & & of nurses experienced in haemodialysis (per $10 \%$ increase) & 0.990 & 0.170 \\
\hline \multirow[t]{8}{*}{$\mathrm{CCl}$} & \multirow[t]{4}{*}{$0,1,2$} & Total number of doctors who provided haemodialysis (per 10-doctor increase) & 1.008 & 0.6615 \\
\hline & & Proportion of haemodialysis patient care specialists (per $10 \%$ increase) & 0.987 & 0.002 \\
\hline & & Total number of nurses who provided haemodialysis (per 10-nurse increase) & 0.889 & 0.000 \\
\hline & & Proportion of nurses experienced in haemodialysis (per 10\% increase) & 0.993 & 0.320 \\
\hline & \multirow[t]{4}{*}{3,4} & Total number of doctors who provided haemodialysis (per 10-doctor increase) & 0.998 & 0.937 \\
\hline & & Proportion of haemodialysis patient care specialists (per 10\% increase) & 0.987 & 0.003 \\
\hline & & Total number of nurses who provided haemodialysis (per 10-nurse increase) & 0.856 & $<0.000$ \\
\hline & & Proportion of nurses experienced in haemodialysis (per 10\% increase) & 0.989 & \\
\hline
\end{tabular}

${ }^{*}$ This table shows the results of subgroup analyses of the relationship between human resources and the risk of hospitalisation according to the presence of a nephrologist, duration of illness and $\mathrm{CCl}$. In this analysis, we adjusted variables such as age, sex, type of insurance coverage, experience of prehospitalisation within 1 year, duration of illness, $\mathrm{CCl}$, type of medical institution, presence of a nephrologist, haemodialysis volume per doctor, number of beds, emergency equipment in the haemodialysis unit, fulfilment rate of criteria for duration of water analysis, number of haemodialysis machines and proportion of medical cost due to CKD. We marked the results with statistically significant values using shadowing.

$\mathrm{CCl}$, Charlson Comorbidity Index; CKD, chronic kidney disease; RR, relative risk.

The prevalence of hypertension and diabetes, along with their associated diseases such as CKD, has gradually increased. ${ }^{15}{ }^{16}$ Concerns regarding the quality of care in managing patients with CKD have been raised, and to solve and prevent these issues, the South Korean government introduced healthcare quality assessment for human and medical resources regarding haemodialysis. ${ }^{10}$ Nevertheless, the reduction of quality of care and the worsening status of patients diagnosed with CKD continue to be debated. Therefore, we set out to investigate the relationship between hospital resources, particularly human resources, and the risk of hospitalisation in patients with ESRD, which was assumed to be indicative of worsening patient status due to a reduction in quality of care.

Our findings suggest that outpatient care for haemodialysis at haemodialysis units with superior human resources, such as a higher proportion of haemodialysis patient care specialists and a higher total number of nurses who provide haemodialysis, was positively associated with better health outcomes in managing patients with CKD needing haemodialysis. Although previous studies show similar results to those reported in this study, such as better outcomes being associated with better haemodialysis unit resources, ours is one of only a small number of studies conducted after the introduction of healthcare quality assessment. ${ }^{17-19}$

In South Korea, HIRA has evaluated the structure, process and outcome indicators of haemodialysis for each haemodialysis unit through healthcare quality assessment, and has provided adjustment payments to medical institutions that were placed in the upper $10 \%$ or were of lower quality based on the evaluation results from 2009. Given the positive impact of superior human resources on patient outcomes as shown in our study, our findings provide helpful information for healthcare quality assessment of haemodialysis due to CKD. However, we found that the total number of nurses who provided haemodialysis had a positive association with patient outcomes in this study, despite its exclusion from the evaluation criteria used in healthcare quality assessment. Therefore, on the basis of our results, health policymakers should consider these criteria in healthcare quality assessment, as well as the weight of each factor during evaluation. In addition, vulnerable patient groups, such as those receiving medical aid and the elderly, were negatively associated with healthcare outcomes after haemodialysis due to ESRD, suggesting an imbalance between the ability to pay and copayment of healthcare services. ${ }^{20}$ Similarly, healthcare professionals 
also assert a need to relax copayment for vulnerable populations. ${ }^{21}$ Thus, support for vulnerable populations should be afforded careful consideration by health policymakers. There was also an inverse association between the proportion of medical cost due to CKD and the risk of hospitalisation, which may have been due to the specialty differences of each medical institution. Similar results were obtained in previous studies, suggesting a positive function for specialty hospitals in South Korea. ${ }^{22}$ Therefore, healthcare professionals should consider increasing their specialties, and policymakers should consider designating additional specialty hospitals for haemodialysis.

Our subgroup analysis also showed interesting results related to efficiency issues. There were several differences in the relationship between human resources and the risk of hospitalisation after haemodialysis due to ESRD. The positive impact of better human resources was greater in patients with a more severe condition, as evidenced by CCI. Thus, the worsening status of a severely ill patient could be more effectively managed by better haemodialysis unit characteristics, such as better staffing. However, the role of each human resource differed at each haemodialysis unit on subgroup analyses according to the presence of a nephrologist. These results suggest that a haemodialysis patient care specialist could be a viable alternative if a haemodialysis unit does not have a nephrologist. Therefore, the importance of professional manpower is also effectively evaluated in establishing a health policy regarding the management of patients with CKD. ${ }^{23}$

These findings have many strengths compared with previous studies. First, the data used in this study were NHI claim data, which included all patients with ESRD in South Korea who received haemodialysis during a 1-year period. Thus, our findings have external validity and would most likely be helpful in establishing evidence-based health policies related to CKD. Second, to the best of our knowledge, ours is the first South Korean study to investigate the relationship between human resources, such as haemodialysis patient care specialists and nurses experienced in haemodialysis, and the risk of hospitalisation as indicators of health outcomes in patients with ESRD receiving haemodialysis after the introduction of healthcare quality assessment for haemodialysis. Therefore, our results may prove useful for designing an effective strategy for managing patients with CKD receiving haemodialysis. Third, we included both patient and haemodialysis unit characteristic variables in this study. Therefore, this study reflects the variety and severity of each patient and the medical institution situation, reducing the limitations of secondary data.

Our study also has several limitations. First, in managing patients with ESRD who needed haemodialysis, there are many factors, including the type of vascular access, laboratory data (eg, serum albumin, haemoglobin), adherence to medical therapy (particularly haemodialysis sessions), more detailed quality indicators and clinical conditions, which can also affect the outcomes of patients with CKD according to previous studies. ${ }^{24-27}$ However, we were unable to include these factors in this study, as the data used were secondary data from the NHI claim data. In addition, detailed information, such as procedures or medications provided to patients with haemodialysis, was not included in this study. Moreover, owing to the nature of the claim data, we could not identify whether hospitalisation was due to haemodialysis, as each hospitalisation was recorded based on the major diagnosis. Therefore, although we assumed that a patient who received haemodialysis progressed to hospitalisation due to ESRD, this hospitalisation might have been caused by a worsening status after haemodialysis. Therefore, more detailed studies are needed. Second, the study period was relatively short (1 year), and we excluded patients with an illness duration of $<1$ year, as patients in the early stages of ESRD could have been hospitalised due to arteriovenous fistula formation from haemodialysis. These problems arose from difficulties in accessing patient information due to primarily ethical issues, as we needed to effectively extract patient information samples from the NHI data. Unfortunately, we were unable to obtain such details. Therefore, to avoid including uncertain causes of hospitalisation (eg, worsening status, arteriovenous fistula formation), we excluded these patients from this study. Third, we considered human resources as independent variables of major interest in this study; however, we could not identify the specific human resources used in the treatment of each patient at each haemodialysis unit due to data limitations. Fourth, to reflect the hospital and patient characteristics in this study, we included variables such as structural characteristics and severity indicators of each patient. Nevertheless, there were several other factors that could have affected the risk of hospitalisation. However, we were unable to consider more detailed variables due to data limitations. Finally, in South Korea, the criteria for physicians who were permitted to provide haemodialysis contrasted with the regulations of certain other countries that permit only nephrologists to perform haemodialysis. Therefore, the relevance of our findings may be limited to South Korea and may not be generalisable to other countries.

Despite the above limitations, our findings suggest that better human resources, such as specialists and nurses experienced in haemodialysis, could positively affect outcomes in patients who receive haemodialysis. In particular, these associations were greater in patients in a clinically vulnerable population, and the role of human resources differed depending on haemodialysis unit characteristics, such as the severity of patients. However, improving hospital staffing to reduce the risk of hospitalisation in such patients would not be an optimal alternative in terms of cost-effectiveness, even if there were many concerns about medical costs related to 
CKD. Therefore, more detailed studies are needed in the future. On the basis of these findings and further studies, health policymakers and healthcare professionals should establish an effective health policy for the appropriate management of patients needing haemodialysis due to CKD.

\section{CONCLUSIONS}

Our findings suggest that outpatient care for haemodialysis at a haemodialysis unit with superior human resources, such as a higher proportion of haemodialysis patient care specialists and a higher number of total nurses who provide haemodialysis, is positively associated with better health outcomes in the management of patients with ESRD receiving haemodialysis. On the basis of our findings, health policymakers and professionals should endeavour to implement strategies for the optimal management of patients with CKD.

\section{Author affiliations}

${ }^{1}$ Department of Health Policy and Management, Graduate School of Public Health, Yonsei University, Seoul, Republic of Korea

${ }^{2}$ Health Insurance Review and Assessment Service, Seoul, Republic of Korea

${ }^{3}$ Department of Public Health, Graduate School, Yonsei University, Seoul, Republic of Korea

${ }^{4}$ Institute of Health Services Research, Yonsei University College of Medicine, Seoul, Republic of Korea

${ }^{5}$ Department of Preventive Medicine, Yonsei University College of Medicine, Seoul, Republic of Korea

${ }^{6}$ National Evidence-Based Healthcare Collaborating Agency, Seoul, Republic of Korea

Contributors $\mathrm{H}-\mathrm{HC}$ and $\mathrm{K}-\mathrm{TH}$ designed the study, performed the research and statistical analyses, and wrote the manuscript. K-TH, CMN, K-TM and E-CP contributed to the Discussion section and reviewed and edited the manuscript. E-CP is the guarantor of this work and, as such, has full access to all the data in the study and takes responsibility for the integrity of the data and the accuracy of the data analysis. The English in this document has been checked by at least two professional editors, both native English speakers. In addition, WK provided re-editing services for our manuscript to improve the quality of the scientific writing.

\section{Competing interests None declared.}

Provenance and peer review Not commissioned; externally peer reviewed.

Data sharing statement No additional data are available.

Open Access This is an Open Access article distributed in accordance with the Creative Commons Attribution Non Commercial (CC BY-NC 4.0) license, which permits others to distribute, remix, adapt, build upon this work noncommercially, and license their derivative works on different terms, provided the original work is properly cited and the use is non-commercial. See: http:// creativecommons.org/licenses/by-nc/4.0/

\section{REFERENCES}

1. Kim EY, Kim CY. Who wants to enter a long-term care facility in a rapidly aging non-western society? Attitudes of older Koreans toward long-term care facilities. J Am Geriatr Soc 2004;52:2114-19.

2. Korea Centers for Disease Control and Prevention. Korea National Health and Nutrition Examination Survey. Secondary Korea National Health and Nutrition Examination Survey 2013. https://knhanes.cdc. go.kr/knhanes/eng/ (accessed online August 2016)

3. Levey AS, Coresh J. Chronic kidney disease. Lancet 2012;379:165-80.
4. Wyld M, Lisa Morton R, Hayen A, et al. A systematic review and meta-analysis of utility-based quality of life in chronic kidney disease treatments. PLoS Med 2012;9:e1001307.

5. Levey AS, Coresh J, Balk E, et al. National Kidney Foundation practice guidelines for chronic kidney disease: evaluation, classification, and stratification. Ann Intern Med 2003;139:137-47.

6. Kim H, Kim KH, Park K, et al. A population-based approach indicates an overall higher patient mortality with peritoneal dialysis compared to hemodialysis in Korea. Kidney Int 2014;86:991-1000.

7. Health Insurance Review \& Assessment Service. Statistics for diseases 2009-13. http://www.hira.or.kr/eng/index.html (accessed online August 2016).

8. Argentero P, Dell'Olivo B, Ferretti MS, et al. Staff burnout and patient satisfaction with the quality of dialysis care. Am J Kidney Dis 2008:51:80-92.

9. Klersy C, Callegari A, Martinelli V, et al. Burnout in health care providers of dialysis service in Northern Italy-a multicentre study. Nephrol Dial Transplant 2007;22:2283-90.

10. Kim SM, Jang WM, Ahn HA, et al. Korean National Health Insurance value incentive program: achievements and future directions. J Prev Med Public Health 2012;45:148.

11. Go AS, Chertow GM, Fan D, et al. Chronic kidney disease and the risks of death, cardiovascular events, and hospitalization. $N$ Engl J Med 2004;351:1296-305.

12. Forman DE, Butler J, Wang $\mathrm{Y}$, et al. Incidence, predictors at admission, and impact of worsening renal function among patients hospitalized with heart failure. J Am Coll Cardiol 2004;43:61-7.

13. Jungers P, Massy ZA, Nguyen-Khoa T, et al. Longer duration of predialysis nephrological care is associated with improved long-term survival of dialysis patients. Nephrol Dial Transplant 2001;16:2357-64.

14. Termorshuizen F, Korevaar JC, Dekker FW, et al. Hemodialysis and peritoneal dialysis: comparison of adjusted mortality rates according to the duration of dialysis: analysis of the Netherlands Cooperative Study on the Adequacy of Dialysis 2. J Am Soc Nephrol 2003;14:2851-60.

15. Ayodele OE, Alebiosu CO. Burden of chronic kidney disease: an international perspective. Adv Chronic Kidney Dis 2010;17:215-24.

16. Couser WG, Remuzzi G, Mendis S, et al. The contribution of chronic kidney disease to the global burden of major noncommunicable diseases. Kidney Int 2011;80:1258-70.

17. Needleman J, Buerhaus $P$, Mattke $S$, et al. Nurse-staffing levels and the quality of care in hospitals. $N$ Engl $\mathrm{J}$ Med 2002;346:1715-22.

18. Thomas-Hawkins C, Flynn L, Clarke SP. Relationships between registered nurse staffing, processes of nursing care, and nurse-reported patient outcomes in chronic hemodialysis units. Nephrol Nurs J 2008;35:123-30;145; quiz 131

19. Gardner JK, Thomas-Hawkins C, Fogg L, et al. The relationship between nurses' perceptions of the hemodialysis unit work environment and nurse turnover, patient satisfaction, and hospitalizations. Nephrol Nurs J 2007;34:271-81; quiz 282.

20. Choi JW, Park EC, Chun SY, et al. Health care utilization and costs among medical-aid enrollees, the poor not enrolled in medical-aid, and the near poor in South Korea. Int J Equity Health 2015;14:128.

21. Kim JH, Lee KS, Yoo KB, et al. The differences in health care utilization between medical aid and health insurance: a longitudinal study using propensity score matching. PLOS ONE 2015;10: e0119939.

22. Kim SJ, Park E-C, Jang SI, et al. An analysis of the inpatient charge and length of stay for patients with joint diseases in Korea: specialty versus small general hospitals. Health Policy 2013:113:93-9.

23. Mandel SR, Martin PL, Blumoff RL, et al. Vascular access in a University transplant and dialysis program. Results, costs, and manpower implications. Arch Surg 1977;112:1375-80.

24. Hsu CY, Ordoñez JD, Chertow GM, et al. The risk of acute renal failure in patients with chronic kidney disease. Kidney Int 2008;74:101-7.

25. Lo LJ, Go AS, Chertow GM, et al. Dialysis-requiring acute renal failure increases the risk of progressive chronic kidney disease. Kidney Int 2009;76:893-9.

26. Tonelli M, Wiebe N, Culleton B, et al. Chronic kidney disease and mortality risk: a systematic review. J Am Soc Nephrol 2006;17:2034-47.

27. Chawla LS, Amdur RL, Amodeo S, et al. The severity of acute kidney injury predicts progression to chronic kidney disease. Kidney Int 2011;79:1361-9. 
Correction: Association between human resources and risk of hospitalisation in end-stage renal disease outpatients receiving haemodialysis: a longitudinal cohort study using claim data during 2013-2014

Choi H-H, Han K-T, Nam CM, et al. Association between human resources and risk of hospitalisation in end-stage renal disease outpatients receiving haemodialysis: a longitudinal cohort study using claim data during 2013-2014. BMJ Open 2016;6:e11319. The following statement should be included in this paper: H-HC and K-TH contributed equally to this work and should be considered co-first authors.

Open Access This is an Open Access article distributed in accordance with the Creative Commons Attribution Non Commercial (CC BY-NC 4.0) license, which permits others to distribute, remix, adapt, build upon this work noncommercially, and license their derivative works on different terms, provided the original work is properly cited and the use is non-commercial. See: http://creativecommons.org/licenses/by-nc/4.0/

BMJ Open 2016;6:e011319corr1. doi:10.1136/bmjopen-2016-011319corr1 